\title{
Free products of pseudocomplemented semilattices - revisited
}

\author{
M. E. AdAMS AND JÜrg SCHMID \\ To Tibor Katriñák on the occasion of his Y0th birthday
}

\begin{abstract}
We analyze the structure of free products of pseudocomplemented semilattices in terms of their skeletons and Glivenko classes by giving a rather explicit construction, complementing the description given by Katriňák and Heleyová in [6].
\end{abstract}

\section{Introduction}

Free products of pseudocomplemented semilattices (which, for brevity, we will refer to as p-semilattices) have been described by Katriňák and Heleyová in [6], giving an ingenious but intricate construction. Recently, the present authors had to construct explicitly a couple of very specific free products of p-semilattices in their pursuit of establishing relative (to Boolean algebras) universality of the category of all p-semilattices (see [1]). It became apparent that an approach to free products focussing on the structure of their Glivenko classes should work quite generally, generalizing the construction of free psemilattices given by the second-named author in [8] and [9]. The purpose of this note is to provide a description of free products of p-semilattices which, we believe, is rather transparent. Moreover, it reduces directly to the the description of free p-semilattices cited above when forming free products of copies of the 1-generated free p-semilattice (the pentagon $N_{5}$ ). Terminology and notation is standard; for unexplained notions specific to p-semilattices, the reader is referred to [3, Chapter 3$]$.

\section{Set-up}

Let $\mathbf{S}_{i}=\left(S_{i} \mid \wedge,{ }^{*}, 0,1\right)$ for $i \in I$ be p-semilattices with associated order $\leq$. If necessary, operations, constants and order will be subscripted with $i$; PCS denotes the class of all p-semilattices. Define the skeleton of $S_{i}$ to be $S k\left(S_{i}\right)=\left\{\xi \in S_{i} \mid \xi=\xi^{* *}\right\}=\left\{\xi \in S_{i} \mid \xi=\eta^{*}\right.$ for some $\left.\eta \in S_{i}\right\}$.

Presented by R. Freese.

Received April 25, 2009; accepted in final form January 27, 2010.

2000 Mathematics Subject Classification: Primary: 06A12; Secondary: 08A10, 08B20.

Key words and phrases: pseudocomplemented semilattice, skeleton, Glivenko class, free products.

While working on this paper, the first author was supported by US CRDF grant KYM12852-BI-07 and the second by Swiss NSF grant 200020-117840/1. 
Defining $\xi \underline{\vee} \eta=\left(\xi^{*} \wedge \eta^{*}\right)^{*}$ for $\xi, \eta \in S k\left(S_{i}\right)$, we obtain a Boolean algebra $S k\left(\mathbf{S}_{i}\right)=\left(S k\left(S_{i}\right) \mid \wedge, \underline{\vee},{ }^{*}, 0,1\right)$. Since the $\left(\wedge,{ }^{*}, 0,1\right)$-reduct of any Boolean algebra is a p-semilattice, $S k\left(\mathbf{S}_{i}\right)$ becomes a sub-p-semilattice of $\mathbf{S}_{i}$ by dropping $\checkmark$ from its type. Elements of any skeleton will also be called Boolean. Denote the class of all Boolean algebras by BA. For notational convenience, we will use lower case Roman letters to denote Boolean elements whenever feasible, while lower case Greek letters will stand for any elements in p-semilattices (Boolean or not).

Let $\xi \in S_{i}$. We write $\Gamma_{i}(\xi)$ for $\left\{\eta \in S_{i} ; \eta^{* *}=\xi^{* *}\right\}$, the Glivenko class of $\xi$ in $S_{i}$. Each Glivenko class contains a unique Boolean element $a_{i} \in S k\left(S_{i}\right)$, its greatest element under the induced order, and $\Gamma\left(a_{i}\right)=\left\{\xi_{i} \in S_{i} \mid \xi_{i}^{* *}=a_{i}\right\}$. Moreover, the Glivenko map $g_{i}: \mathbf{S}_{i} \rightarrow S k\left(\mathbf{S}_{i}\right)$ sending $\xi_{i} \in S_{i}$ to $\xi_{i}^{* *} \in S k\left(S_{i}\right)$ is a surjective PCS-homomorphism. Note also that any PCS-homomorphism between between Boolean algebras is a BA-homomorphism, making BA a full subcategory of PCS. The following simple fact is crucial for our purposes:

Fact 2.1. Any PCS-homomorphism $f$ from a p-semilattice $\mathbf{S}$ into any Boolean algebra $\mathbf{B}$ factors through the Glivenko map by $f=f\lceil S k(S) \circ g$.

Proof. Assume $f: \mathbf{S} \rightarrow \mathbf{B}$ is a PCS-homomorphism. Then for $\xi \in S$ one has $f(\xi)=f(\xi)^{* *}$ as $\mathbf{B}$ is Boolean, and thus $f(\xi)=f\left(\xi^{* *}\right)=f(g(\xi))$. It follows that $f=f\lceil S k(S) \circ g$.

For a family of p-semilattices $\mathbf{S}_{i}(i \in I)$, a PCS-free product consists of a p-semilattice $\mathbf{S}$ together with a family $\left\{e_{i}: \mathbf{S}_{i} \rightarrow \mathbf{S}\right\}$, for $i \in I$, of PCSembeddings, subject to the following two conditions: For any p-semilattice $\mathbf{T}$ and any family of PCS-homomorphisms $t_{i}: \mathbf{S}_{i} \rightarrow \mathbf{T}(i \in I)$, there exists a homomorphism $t: \mathbf{S} \rightarrow \mathbf{T}$ such that $t_{i}=t \circ e_{i}$ for all $i \in I$ and $\mathbf{S}$ is generated by $\bigcup_{i \in I} e_{i}\left(S_{i}\right)$. It follows that $\mathbf{S}$ is unique up to isomorphism whenever it exists, and will be denoted by $\coprod_{\mathbf{P C S}}\left\{\mathbf{S}_{i} \mid i \in I\right\}$ in the sequel.

The BA-free product of any family $\left\{\mathbf{B}_{i} \mid i \in I\right\}$ of Boolean algebras, denoted by $\coprod_{\mathbf{B A}}\left\{\mathbf{B}_{i} \mid i \in I\right\}$, is defined similarly, using BA-homomorphisms.

The existence of PCS-free products may be deduced from very general theorems (see, e.g., [4, Section 29]), but our primary aim here is to provide a transparent explicit construction. So we only dispose of a trivial case at this point: If one of the p-semilattices $\mathbf{S}_{i}$ is the one-element algebra $\mathbf{1}$, then $\coprod_{\mathbf{P C S}}\left\{\mathbf{S}_{i} ; i \in I\right\}$ exists if and only if $\mathbf{S}_{i} \cong \mathbf{1}$ for all $i \in I$, and then obviously $\coprod_{\mathbf{P C S}}\left\{\mathbf{S}_{i} \mid i \in I\right\} \cong \mathbf{1}$. This readily follows from the fact that there is no PCS-embedding $e: \mathbf{1} \rightarrow \mathbf{S}$ unless $\mathbf{S} \cong \mathbf{1}$. So we assume henceforth that $\mathbf{S}_{i} \neq 1$ for all $i \in I$ and, w.l.o.g. as we shall see, that $S_{i} \cap S_{j}=\{0,1\}$ for $i \neq j$.

The following result is Proposition 1 of [6]. To make the paper reasonably self-contained, we include a proof adapted to our notational conventions.

Proposition 2.2. (Katriňák and Heleyová [6]) If $\mathbf{S} \cong \coprod_{\mathbf{P C S}}\left\{\mathbf{S}_{i} \mid i \in I\right\}$, then $S k(\mathbf{S}) \cong \coprod_{\mathbf{B A}}\left\{S k\left(\mathbf{S}_{i}\right) \mid i \in I\right\}$. 
Proof. Consider $\mathbf{C} \in \mathbf{B A}$ and a family $\left\{f_{i}: S k\left(\mathbf{S}_{i}\right) \rightarrow \mathbf{C}\right\}$, for $i \in I$, of Boolean homomorphisms. With $g_{i}: \mathbf{S}_{i} \rightarrow S k\left(\mathbf{S}_{i}\right)$ the Glivenko maps, we define $f_{i}^{\prime}:=f_{i} \circ g_{i}: \mathbf{S}_{i} \rightarrow \mathbf{C}$ for $i \in I$. Since $\mathbf{S} \cong \coprod_{\mathbf{P C S}}\left\{\mathbf{S}_{i} \mid i \in I\right\}$, there exists a PCS-homomorphism $t: \mathbf{S} \rightarrow \mathbf{C}$ such that $f_{i}^{\prime}=t \circ e_{i}$ for all $i \in I$. Now $t=t\lceil S k(\mathbf{S}) \circ g$ by Fact 2.1, where $g$ is the Glivenko map $g: \mathbf{S} \rightarrow S k(\mathbf{S})$; hence $f_{i} \circ g_{i}=f_{i}^{\prime}=t\left\lceil S k(\mathbf{S}) \circ g \circ e_{i}\right.$. Let $\xi \in S k\left(\mathbf{S}_{i}\right)$. Then $g_{i}(\xi)=\xi$ and $g\left(e_{i}(\xi)\right)=e_{i}(\xi)$, so that $f_{i}=t\left\lceil S k(\mathbf{S}) \circ e_{i} \uparrow S k\left(\mathbf{S}_{i}\right)\right.$ for all $i \in I$. Both $t \uparrow S k(\mathbf{S})$ and $e_{i} \uparrow S k\left(\mathbf{S}_{i}\right)$ are BA-homomorphisms as PCS-homomorphisms between Boolean algebras; so $t\lceil S k(\mathbf{S})$ is the map required for $S k(\mathbf{S})$ to be a free product of the $S k\left(\mathbf{S}_{i}\right)$.

Since $\mathbf{S}=\left[\bigcup_{i \in I} e_{i}\left(S_{i}\right)\right]$, where $\left[\bigcup_{i \in I} e_{i}\left(S_{i}\right)\right]$ denotes the subalgebra generated by $\bigcup_{i \in I} e_{i}\left(S_{i}\right)$, we have $S k(\mathbf{S}) \subseteq\left[\bigcup_{i \in I} e_{i}\left(S_{i}\right)\right]$. An induction on the length of $\xi \in S$ shows that for any $\xi$ such that $\xi=\xi^{* *}$, we have $\xi \in\left[\bigcup_{i \in I} e_{i}\left(S k\left(S_{i}\right)\right)\right]$. To see this, consider $\xi$ for which $\xi=\xi^{* *}$. If $\xi=\alpha^{*}$, then either $\alpha=\beta^{*}$ or $\alpha=\beta \wedge \gamma$. If $\alpha=\beta^{*}$, then (since $\beta^{* * *}=\beta^{*}$ in any p-semilattice) $\xi=\alpha^{*} \in$ $\left[\bigcup_{i \in I} e_{i}\left(S k\left(S_{i}\right)\right)\right]$. If $\alpha=\beta \wedge \gamma$, then $\alpha^{*}=(\beta \wedge \gamma)^{*}=(\beta \wedge \gamma)^{* * *}=\left(\beta^{* *} \wedge \gamma^{* *}\right)^{*}$ (since $(\beta \wedge \gamma)^{* *}=\beta^{* *} \wedge \gamma^{* *}$ in any p-semilattice) and, since the lengths of $\beta^{*}$ and $\gamma^{*}$ are less than that of $\alpha$, again $\xi=\alpha^{*} \in\left[\bigcup_{i \in I} e_{i}\left(S k\left(S_{i}\right)\right)\right]$. If, alternatively, $\xi=\alpha \wedge \gamma$, then $\xi=\xi^{* *}=(\alpha \wedge \beta)^{* *}=\alpha^{* *} \wedge \beta^{* *}$ and, since the lengths of $\alpha^{*}$ and $\beta^{*}$ are less than that of $\xi$, as before $\xi=\alpha \wedge \gamma \in\left[\bigcup_{i \in I} e_{i}\left(S k\left(S_{i}\right)\right)\right]$. That is, as required, $S k(\mathbf{S}) \subseteq\left[\bigcup_{i \in I} e_{i}\left(S k\left(S_{i}\right)\right)\right]$.

\section{The Boolean case}

BA-free products of Boolean algebras may be described in different ways, for a purely algebraic approach see, e.g., [7, Chapter 4]. In order to facilitate the introduction of so-called upper covers - see below - and for the simplicity of the resulting construction, we adopt in this section an approach based on Stone duality, see, e.g., [10, Section 13].

Let $\left\{\mathbf{B}_{i} \mid i \in I\right\}$ be any family of Boolean algebras, and $\mathbf{X}_{i}=\left(X_{i} ; \tau_{i}\right)$ the Stone space associated to $\mathbf{B}_{i}$ for $i \in I$. That is, the topology $\tau_{i}$ is Hausdorff, compact and totally disconnected, and $\mathbf{B}_{i}$ can be recovered from $\mathbf{X}_{i}$ as the collection of all clopen subsets of $\mathbf{X}_{i}$ endowed with set theoretic operations of union, intersection and complement, and $\emptyset$, respectively $X_{i}$, as constants. Conversely, $\mathbf{X}_{i}$ may be constructed as the space of all ultrafilters of $\mathbf{B}_{i}$ endowed with the hull-kernel topology, but this is not needed here. Moreover, $\mathbf{X}_{i}$ and $\mathbf{B}_{i}$ determine each other up to homeomorphism, respectively (Boolean) isomorphism.

Let $\mathbf{X}=(X ; \tau):=\prod_{i \in I} \mathbf{X}_{i}$ be the topological product of the Stone spaces of the Boolean algebras $\mathbf{B}_{i}$. It is well-known that $\mathbf{X}$ is the Stone space of $\mathbf{B}=\coprod_{\mathbf{B A}}\left\{\mathbf{B}_{i} \mid i \in I\right\}$ (up to homeomorphism). In particular, a subset $U \subseteq X$ is associated with an element of $B$ if and only if $U$ is a clopen subset of $X$ if and only if $U=\emptyset$ or $U$ may be written (non-uniquely) in so-called normal form as a finite union $U=U_{1} \cup \cdots \cup U_{n}$ where, for $1 \leq j \leq n, U_{j}=\prod_{i \in I} U_{j i}$ 
such that $\emptyset \neq U_{j i} \subseteq X_{i}$ is clopen and $U_{j i} \neq X_{i}$ for at most finitely many $i \in I$. The required $\mathbf{B A}$-embeddings $e_{i}: \mathbf{B}_{i} \rightarrow \mathbf{B}$ are provided for all $j \in I$ by $e_{i}\left(V_{i}\right)=\pi_{i}^{-1}\left(V_{i}\right)=V_{i} \times \prod_{j \in I}\left(X_{i} \mid j \neq i\right)$ for a clopen subset $V_{i} \subseteq X_{i}$, where $\pi_{i}$ is the canonical projection of $\mathbf{X}$ onto $\mathbf{X}_{i}$. It follows that $U$ in normal form is a finite union of finite intersections of nonempty sets of type $e_{i}\left(V_{i}\right)$. Obviously, for $U_{j}$ as above, one has

$$
U_{j} \subseteq e_{i}\left(V_{i}\right) \text { for some clopen } V_{i} \subseteq X_{i} \text { iff } V_{i} \supseteq U_{j i}
$$

Writing $\mathbf{B}_{i}^{\prime}$ for the canonical copy $e_{i}\left(\mathbf{B}_{i}\right)$ of $\mathbf{B}_{i}$ within $\mathbf{B}$, we define maps $\mathrm{c}_{i}: \mathbf{B} \rightarrow \mathbf{B}_{i}^{\prime}$, for all $i \in I$, by $\mathrm{c}_{i}(U):=\pi_{i}^{-1} \circ \pi_{i}(U)$ for any clopen subset $U \subseteq X$. It is immediate that $c_{i}$ distributes over finite unions (but not necessarily over finite intersections), hence $c_{i}$ is order-preserving. If $U$ is given in canonical form $U=U_{1} \cup \cdots \cup U_{n}$, then $\mathrm{c}_{i}(U)=\mathrm{c}_{i}\left(U_{1}\right) \cup \cdots \cup \mathrm{c}\left(U_{n}\right)=U_{1 i} \cup \cdots \cup U_{n i}$; thus $\mathrm{c}_{i}(U)$ is the smallest member of $\mathbf{B}_{i}^{\prime}$ containing $U$ by (3.1). It also follows from (3.1) that for any clopen $\emptyset \neq U \subseteq X$, one has $c_{i}(U) \neq X_{i}$ for at most finitely many $i \in I$.

Reversing the process, we consider a finite subset $I_{\text {fin }}=\left\{i_{1}, \ldots, i_{m}\right\} \subseteq I$ and clopen subsets $\emptyset \neq V_{i} \subsetneq X_{i}$ for $i \in I_{\text {fin }}$, looking for clopen subsets $U \subseteq X$ such that $\mathrm{c}_{i}(U)=V_{i}$ for $i \in I_{\text {fin }}$ and $\mathrm{c}_{i}(U)=X_{i}$ for $i \notin I_{\text {fin }}$. Writing $U$ in normal form, this amounts to requiring $U_{1 i} \cup \cdots \cup U_{n i}=V_{i}$ for $i \in I_{\text {fin }}$ and $U_{1 i} \cup \cdots \cup U_{n i}=X_{i}$ for $i \notin I_{\text {fin }}$. It follows that there is a unique largest such $U$, given by $U_{\max }:=\prod_{i \in I}\left(W_{i} \mid W_{i}=V_{i}\right.$ for $i \in I_{\text {fin }}$ and $W_{i}=X_{i}$ otherwise $)$.

Leaving Stone duality here for good, we will identify $\mathbf{B}_{i}$ and $\mathbf{B}_{i}^{\prime}$ henceforth and think of $\mathbf{c}_{i}$ as a map from $\mathbf{B}$ into $\mathbf{B}_{i}$. So for $b \in B, c_{i} b$ is the smallest element $x \in B_{i}$ such that $x \geq b$. For notational convenience, we will mostly write $b^{(i)}$ for $c_{i} b$, for all $b \in B$ and $i \in I$, and call $b^{(i)}$ the upper $i$-cover of $b$. By the above arguments, $c_{i}$ is a join-homomorphism, and thus order-preserving, but not a meet-homomorphism in general. Moreover, the following is a direct consequence of (3.1).

Fact 3.1. For $0 \neq b \in B, c_{i} b \neq 1_{i}$ for at most finitely many $i \in I$.

Note also that by replacing $\mathbf{B}_{i}$ with $\mathbf{B}_{i}^{\prime}$ we identify the zeros $0_{i}$, respectively the units $1_{i}$, of the algebras $\mathbf{B}_{i}$ with the zero 0 , respectively the unit 1 , of $\mathbf{B}$ - which is the main reason for postulating that $S_{i} \cap S_{j}=\{0,1\}$ for $i \neq j$ at the end of section 2. However, we keep the subscripts on 0 and 1 whenever it seems desirable to track the history of such an element.

Any $b \in B$ may be written (non-uniquely) in disjunctive normal form, that is, as $b=b_{1} \vee \cdots \vee b_{n}$ where each $b_{j}(1 \leq j \leq n)$ has the form $b_{j}=\bigwedge_{i \in I_{j}} b_{i}^{j}$ for some finite subset $I_{j} \subseteq I$ with $b_{i}^{j} \in B_{i} \backslash\left\{0_{i}\right\}$ for $i \in I_{j}$. Equation (3.1) above takes the form

$$
b_{j} \leq a_{i} \text { for } a_{i} \in B_{i} \text { iff } a_{i}=1_{i} \text { or }\left(i \in I_{j} \text { and } a_{i} \geq b_{i}^{j}\right) .
$$


It follows that $c_{i} b_{j}=b_{j}^{(i)}=b_{i}^{j}$ iff $i \in I_{j}$ and $c_{i} b_{j}=1_{i}$ iff $i \notin I_{j}$, and thus $c_{i} b=c_{i} b_{1} \vee \cdots \vee c_{i} b_{n}$ equals either $b_{i}^{1} \vee \cdots \vee b_{i}^{n}\left(\right.$ if $\left.i \in I_{1} \cap \cdots \cap I_{n}\right)$ or $1_{i}$ (otherwise).

We have thus an algebraic version for the construction of elements with prescribed $i$-covers:

Fact 3.2. Let $I_{\text {fin }}=\left\{i_{1}, \ldots, i_{m}\right\} \subseteq I$ be finite and $0 \neq a_{i} \in B_{i}$ for $i \in I_{\text {fin }}$. Then there exists a unique largest $b \in B$ such that $b^{(i)}=a_{i}$ for $i \in I_{\text {fin }}$ and $b^{(i)}=1_{i}$ for $i \notin I_{\text {fin }}$, given explicitly as $b_{\max }=a_{i_{1}} \wedge \cdots \wedge a_{i_{m}}$. More generally, $b \in B$ satisfies $b^{(i)}=a_{i}$ for $i \in I_{\text {fin }}$ and $b^{(i)}=1_{i}$ for $i \notin I_{\text {fin }}$ iff $b$ has a disjunctive normal form $b=b_{1} \vee \cdots \vee b_{n}$ such that $I_{\text {fin }}=I_{1} \cap \cdots \cap I_{n}$ and $a_{i}=b_{i}^{1} \vee \cdots \vee b_{i}^{n}$ for $i \in I_{\text {fin }}$.

\section{The Construction}

Our plan is as follows: Suppose for the moment that $\mathbf{S}=\coprod_{\mathbf{P C S}}\left\{\mathbf{S}_{i} \mid i \in I\right\}$ exists, and let $\mathbf{B}:=S k(\mathbf{S})$ as well as $\mathbf{B}_{i}:=S k\left(\mathbf{S}_{i}\right)(i \in I)$ in this section. Then we know by Proposition 2.2 that $\mathbf{B}=\coprod_{\mathbf{B A}}\left\{\mathbf{B}_{i} \mid i \in I\right\}$; so we aim to describe the members of $\Gamma(b)$ for any $b \in B$. Since $S$ is generated by $\bigcup_{i \in I} S_{i}$, these members should be finite meets (taken within $\mathbf{S}$ ) of type $b \wedge \xi_{i_{1}} \wedge \cdots \wedge \xi_{i_{n}}$ with $b \in B$ and $\xi_{i_{k}} \in S_{i_{k}}$. We need not be concerned with pseudocomplements here since $\eta^{*} \in B$ for any $\eta \in S$.

Looking at the simplest case, consider $b \wedge \xi \in \Gamma(b)$ with $b \in B$ and $\xi \in S_{i}$. Since $b \leq b^{(i)}$, we have $b \wedge \xi=b \wedge \xi^{\prime}$ where $\xi^{\prime}=b^{(i)} \wedge \xi \in S_{i}$. Now $\xi^{* *} \leq$ $\left(b^{(i)}\right)^{* *}=b^{(i)}$; on the other hand, $\xi^{* *} \geq\left(b \wedge \xi^{\prime}\right)^{* *}=b$ (with the last equality holding since $\left.b \wedge \xi^{\prime}=b \wedge \xi \in \Gamma(b)\right)$. As $b^{(i)}$ is the upper $i$-cover of $b$, we conclude $\xi^{\prime * *}=b^{(i)}$. It follows that any meet $b \wedge \xi \in \Gamma(b)$ with $\xi \in S_{i}$ may be written as $b \wedge \xi^{\prime}$ with $\xi^{\prime} \in \Gamma_{i}\left(b^{(i)}\right)$. This motivates the following definition:

Definition 4.1. For $b \in B$, let $\Lambda(b)$ be the collection of all $\beta=\left(\beta_{i}\right) \in \prod_{i \in I} S_{i}$ such that $\beta_{i} \in \Gamma_{i}\left(b^{(i)}\right)$ for all $i \in I$ but $\beta_{i} \neq b^{(i)}$ for at most finitely many $i \in I$. Put $P(b):=\{(b ; \beta) ; \beta \in \Lambda(b)\}$, and let $P:=\bigcup_{b \in B} P(b)$.

Remark 4.2. It may help to think of the members of $P$ as coding up the hypothetical meets (in $\mathbf{S}$ ) of type $b \wedge \xi_{i_{1}} \wedge \cdots \wedge \xi_{i_{n}}$ with $\xi_{i_{k}} \in S_{i_{k}}$, as considered above (recall Fact 3.1). This will be made precise by Corollary 4.9.

Notation and nomenclature 4.3. Elements of $P$ will be written as $\boldsymbol{\beta}=$ $(b ; \beta)=\left(b ;\left(\beta_{i}\right)\right)$, and analogously for other lower-case roman and corresponding greek letters. We call the Boolean element $b \in B$ the head and the $\left.\beta_{i} \in \Gamma_{i}\left(b^{(i)}\right)\right)$ the components of $\boldsymbol{\beta}$; the latter are also referred to by $(\boldsymbol{\beta})_{i}:=\beta_{i}$.

As $P \subseteq B \times \prod_{i \in I} S_{i}$, the product order on $B \times \prod_{i \in I} S_{i}$ induces an order $\leq_{P}$ on $P$. The main result of this paper (Theorem 4.10) is that $\leq_{P}$ induces the structure of a pseudocomplemented semilattice on $\left(P ; \leq_{P}\right)$ which makes it a $\mathbf{P C S}$-free product of the $\mathbf{S}_{i}$. 
Lemma 4.4. $\left(P ; \leq_{P}\right)$ is a meet-semilattice with least element $0_{P}:=\left(0 ;\left(0_{i}\right)\right)$ and greatest element $1_{P}:=\left(1 ;\left(1_{i}\right)\right)$. The meet of $\boldsymbol{\alpha}=\left(a ;\left(\alpha_{i}\right)\right), \boldsymbol{\beta}=\left(b ;\left(\beta_{i}\right)\right)$ in $\left(P ; \leq_{P}\right)$ is given by $\boldsymbol{\alpha} \wedge_{P} \boldsymbol{\beta}:=\left(a \wedge b ;\left(\alpha_{i} \wedge \beta_{i} \wedge(a \wedge b)^{(i)}\right)\right)$.

Proof. Consider $\boldsymbol{\gamma}=\left(c ;\left(\gamma_{i}\right)\right) \in P$ such that $\boldsymbol{\gamma} \leq \boldsymbol{\alpha}, \boldsymbol{\beta}$. The definition of $\leq_{P}$ gives $c \leq a \wedge b$ and $\gamma_{i} \leq \alpha_{i} \wedge \beta_{i}$. Since $\gamma \in P$, one also has $\gamma_{i}^{* *}=c^{(i)}$, thus $\gamma_{i} \leq \gamma_{i}^{* *}=c^{(i)} \leq(a \wedge b)^{(i)}$ and so $\gamma_{i} \leq \alpha_{i} \wedge \beta_{i} \wedge(a \wedge b)^{(i)}$. It remains to show that $\left(a \wedge b ;\left(\alpha_{i} \wedge \beta_{i} \wedge(a \wedge b)^{(i)}\right)\right)$ actually is in $P$. But $\left(\alpha_{i} \wedge \beta_{i} \wedge(a \wedge b)^{(i)}\right)^{* *}=$ $\alpha_{i}^{* *} \wedge \beta_{i}^{* *} \wedge\left((a \wedge b)^{(i)}\right)^{* *}=a^{(i)} \wedge b^{(i)} \wedge(a \wedge b)^{(i)}=(a \wedge b)^{(i)}$ as $(a \wedge b)^{(i)} \leq a^{(i)} \wedge b^{(i)}$ (since $c_{i}$ is order-preserving).

The subscripts $P$ on $\leq, \wedge, 0$ and 1 may be dropped henceforth, since, hopefully, there is no danger of confusion.

Lemma 4.5. Let $(P ; \wedge, 0,1)$ be the semilattice of Lemma 4.4. Then $(P ; \wedge, 0,1)$ is a pseudocomplemented semilattice, the pseudocomplement of $\boldsymbol{\alpha}=\left(a ;\left(\alpha_{i}\right)\right)$ being given by $\boldsymbol{\alpha}^{* P}=\left(a^{*} ;\left(\left(a^{*}\right)^{(i)}\right)\right)$.

Proof. Fix $\boldsymbol{\alpha}=\left(a ;\left(\alpha_{i}\right)\right)$ and suppose $\boldsymbol{\alpha} \wedge \boldsymbol{\gamma}=0$ for some $\boldsymbol{\gamma}=\left(c ;\left(\gamma_{i}\right)\right)$. By Lemma 4.4 we have $a \wedge c=0$, that is, $c \leq a^{*}$, and so $\gamma_{i} \leq c^{(i)} \leq\left(a^{*}\right)^{(i)}$. On the other hand, $\left(a ;\left(\alpha_{i}\right)\right) \wedge\left(a^{*},\left(\left(a^{*}\right)^{(i)}\right)\right)=\left(a \wedge a^{*} ;\left(\alpha_{i} \wedge\left(a^{*}\right)^{(i)} \wedge\left(a \wedge a^{*}\right)^{(i)}\right)\right)=$ $\left(0 ;\left(0_{i}\right)\right)$ since $\left(a \wedge a^{*}\right)^{(i)}=0^{(i)}=0_{i}$.

We write $\mathbf{P}$ for the p-semilattice $\mathbf{P}=\left(P ; \wedge,{ }^{*}, 0,1\right)=\left(P ; \wedge_{P},{ }^{*}{ }_{P}, 0_{P}, 1_{P}\right)$.

Define a map $\iota: B \rightarrow P$ by $\iota(a):=\left(a ;\left(a^{(i)}\right)\right)$ for all $a \in B$.

Corollary 4.6. The map $\iota$ is a (Boolean) isomorphism from $\mathbf{B}$ onto $S k(\mathbf{P})$. Accordingly, we will also write $\mathbf{B}^{\prime}:=\iota(\mathbf{B})$ for the skeleton of $\mathbf{P}$.

Proof. By Lemma 4.5, Sk(P) $=\left\{\boldsymbol{\alpha}^{*} \mid \boldsymbol{\alpha} \in P\right\}=\left\{\left(a^{*} ;\left(\left(a^{(i)}\right)^{*}\right)\right) \mid a \in B\right\}=$ $\left\{\left(a ;\left(a^{(i)}\right)\right) \mid a \in B\right\}$ since $^{*}$ acts bijectively on $B$, respectively $B_{i}$, so $\iota$ is bijective. Let $a, b \in B$, then

$$
\begin{aligned}
\iota(a) \wedge \iota(b) & =\left(a ;\left(a^{(i)}\right)\right) \wedge\left(b ;\left(b^{(i)}\right)\right)=\left(a \wedge b ;\left(a^{(i)} \wedge b^{(i)} \wedge(a \wedge b)^{(i)}\right)\right) \\
& =\left(a \wedge b ;\left((a \wedge b)^{(i)}\right)\right)=\iota(a \wedge b),
\end{aligned}
$$

since $(a \wedge b)^{(i)} \leq a^{(i)} \wedge b^{(i)}$, and $(\iota(a))^{*}=\left(a ;\left(a^{(i)}\right)\right)^{*}=\left(a^{*} ;\left(\left(a^{*}\right)^{(i)}\right)\right)=\iota\left(a^{*}\right)$. Since Boolean join on $\mathbf{B}$, respectively $S k(\mathbf{P})$, is defined in terms of $\wedge$ and ${ }^{*}$, it is also preserved.

Define, for each $j \in I$, a map $\mathrm{e}_{j}: S_{j} \rightarrow P$ by $\mathrm{e}_{j}(\xi):=\left(\xi^{* *} ;\left(\xi_{i}\right)\right)$ for $\xi \in S_{j}$, where $\xi_{i}=\left(\xi^{* *}\right)^{(i)}($ if $i \neq j)$ and $\xi_{i}=\xi$ (if $\left.i=j\right)$. Explicitly, $\mathrm{e}_{j}(\xi)=\left(0 ;\left(0_{i}\right)\right)$ if $\xi=0$, and $\mathrm{e}_{j}(\xi)=\left(\xi^{* *} ;(1, \operatorname{dots}, 1, \xi, 1, \ldots, 1)\right)$ with $\xi$ at the $j$-th position if $\xi \neq 0$.

Lemma 4.7. $\mathrm{e}_{j}$ embeds $\mathbf{S}_{j}$ into $\mathbf{P}$ (as p-semilattices) for all $j \in I$, and $\mathrm{e}_{j} \mid B_{j}$ embeds $\mathbf{B}_{j}$ into $\mathbf{B}^{\prime}=S k(\mathbf{P})$ (as Boolean algebras). 
Proof. Consider $\xi, \eta \in S_{j}$ and put $x:=\xi^{* *}, y:=\eta^{* *}$. The heads of $\mathrm{e}_{j}(\xi)$, respectively $\mathrm{e}_{j}(\eta)$, thus are $x$, respectively $y$, while their components are $x^{(i)}$, respectively $y^{(i)}$, for $i \neq j$ and $\xi$, respectively $\eta$, for $i=j$. This shows that $\mathrm{e}_{j}$ is one-to-one.

Similarly, $\mathrm{e}_{j}(\xi \wedge \eta)$ has head $(\xi \wedge \eta)^{* *}=\xi^{* *} \wedge \eta^{* *}=x \wedge y$ and components $(x \wedge y)^{(i)}$ for $i \neq j$, respectively $\xi \wedge \eta$ for $i=j$. Compute $\mathrm{e}_{j}(\xi) \wedge \mathrm{e}_{j}(\eta)$ according to Lemma 4.4 and obtain $x \wedge y$ for its head, $x^{(i)} \wedge y^{(i)} \wedge(x \wedge y)^{(i)}$ for $i \neq j$, respectively $\xi \wedge \eta \wedge(x \wedge y)^{(j)}$ for $i=j$, for its components. If $i \neq j$, then $x^{(i)} \wedge y^{(i)} \wedge(x \wedge y)^{(i)}=(x \wedge y)^{(i)}$ since $c_{i}$ is order-preserving, while $\xi \wedge \eta \wedge(x \wedge y)^{(j)}=\xi \wedge \eta$ since $\xi \wedge \eta \leq x \wedge y \leq(x \wedge y)^{(j)}$. Consequently, $\mathrm{e}_{j}(\xi \wedge \eta)=\mathrm{e}_{j}(\xi) \wedge \mathrm{e}_{j}(\eta)$.

It remains to show that $\mathrm{e}_{j}\left(\xi^{*}\right)=\left(\mathrm{e}_{j}(\xi)\right)^{*}$. According to $4.5,\left(\mathrm{e}_{j}(\xi)\right)^{*}$ has head $x^{*}$ and components $\left(x^{*}\right)^{(i)}$ for all $i \in I$. On the other hand, $\mathrm{e}_{j}\left(\xi^{*}\right)$ has head $\xi^{* * *}=x^{*}$, components $\left(x^{*}\right)^{(i)}$ (if $i \neq j$ ) and $\xi^{*}$ (if $i=j$ ). But $\left(x^{*}\right)^{(j)}=\left(\xi^{* * *}\right)^{(j)}=\left(\xi^{*}\right)^{(j)}=\xi^{*}$ since $\xi^{*} \in B_{j}$, and thus $\mathrm{e}_{j}\left(\xi^{*}\right)=\left(\mathrm{e}_{i}(\xi)\right)^{*}$.

If $\xi \in B_{i}$, then $\xi=\xi^{* *}=x$, and thus $\mathrm{e}_{j}(\xi) \in \mathbf{B}^{\prime}$. As above, Boolean join is preserved automatically.

It follows that $\mathbf{S}_{j}^{\prime}:=\mathrm{e}_{j}\left(\mathbf{S}_{j}\right)$ is a subalgebra of $\mathbf{P}$ isomorphic to $\mathbf{S}_{j}$, and analogously, $\mathbf{B}_{j}^{\prime}:=\mathrm{e}_{j}\left(\mathbf{B}_{j}\right)$ a Boolean subalgebra of $\mathbf{B}^{\prime}$ isomorphic to $\mathbf{B}_{j}$, for all $j \in I$.

Lemma 4.8. (i) The union of all subalgebras $\mathbf{S}_{j}^{\prime}(j \in I)$ generates $\mathbf{P}$ as a p-semilattice.

(ii) The union of all subalgebras $\mathbf{B}_{j}^{\prime}(j \in I)$ generates $\mathbf{B}^{\prime}$ as a Boolean algebra.

Proof. We start with (ii). Consider $b \in B$, thus $b=p\left(b_{j_{1}}, \ldots, b_{j_{n}}\right)$ where $p$ is a Boolean polynomial and $b_{j_{k}} \in B_{j_{k}} \subseteq B$ for $1 \leq k \leq n$. Hence $\iota(b)=$ $\iota\left(p\left(b_{j_{1}}, \ldots, b_{j_{n}}\right)\right)=p\left(\iota\left(b_{j_{1}}\right), \ldots, \iota\left(b_{j_{n}}\right)\right)$. But $\iota\left(b_{j_{k}}\right)=\left(b_{j_{k}} ;\left(b_{j_{k}}^{(i)}\right)\right)=\mathrm{e}_{j_{k}}\left(b_{j_{k}}\right) \in$ $\mathbf{B}_{j_{k}}^{\prime}$ for $1 \leq k \leq n$ and we are done.

For (i), consider $\boldsymbol{\beta}=\left(b ;\left(\beta_{i}\right)\right) \in \mathbf{P}$. It follows from (ii) above that $\boldsymbol{\beta}^{* *}=$ $\left(b ;\left(b^{(i)}\right)\right)$ is in the subalgebra of $\mathbf{P}$ generated by $\bigcup_{j \in I} \mathbf{S}_{j}^{\prime}$. Put $\left\{j_{1}, \operatorname{dot} s, j_{n}\right\}:=$ $\left\{j \in I \mid \beta_{j} \neq b^{(j)}\right\}$. Hence, for $1 \leq k \leq n$, we have $\mathrm{e}_{j_{k}}\left(\beta_{j_{k}}\right)=\left(\xi^{* *} ;\left(\xi_{i}\right)\right)$ with $\xi^{* *}=\left(\beta_{j_{k}}\right)^{* *}=b^{\left(j_{k}\right)} \geq b, \xi_{i}=1$ for $i \neq j_{k}$ and $\xi_{i}=\beta_{j_{k}}$ for $i=j_{k}$. Then $\boldsymbol{\beta}^{* *} \wedge \mathrm{e}_{j_{1}}\left(\beta_{j_{1}}\right) \wedge \cdots \wedge \mathrm{e}_{j_{n}}\left(\beta_{j_{n}}\right)=\left(b ;\left(\eta_{i}\right)\right)$ where $\eta_{i}=b^{(i)}$ for $i \notin\left\{j_{1}, \ldots, j_{n}\right\}$ (the $i$-th components of all $e_{j_{k}}\left(\beta_{j_{k}}\right)$ being 1) while $\eta_{j_{k}}=\beta_{j_{k}} \wedge b^{\left(j_{k}\right)}=\beta_{j_{k}}$ for $i=j_{k}$ with $1 \leq k \leq n$ (the $i$-th components of $\mathrm{e}_{j_{k^{\prime}}}\left(\beta_{j_{k^{\prime}}}\right)$ being 1 for $k^{\prime} \neq k$ ). But this means that $\left(b ;\left(\eta_{i}\right)\right)=\left(b ;\left(\beta_{i}\right)\right)=\boldsymbol{\beta}$, and $\boldsymbol{\beta}$ is in the subalgebra of $\mathbf{P}$ generated by $\bigcup_{j \in I} \mathbf{S}_{j}^{\prime}$.

Corollary 4.9 (Normal forms). For all $\boldsymbol{\beta}=\left(b ;\left(\beta_{i}\right)\right) \in \mathbf{P}$, we have $\boldsymbol{\beta}=$ $\boldsymbol{\beta}^{* *} \wedge \bigwedge_{i \in I} \mathrm{e}_{i}\left(\beta_{i}\right)$.

Proof. In the proof of item (i) just above, we obtained

$$
\boldsymbol{\beta}=\boldsymbol{\beta}^{* *} \wedge \mathrm{e}_{j_{1}}\left(\beta_{j_{1}}\right) \wedge \cdots \wedge \mathrm{e}_{j_{n}}\left(\beta_{j_{n}}\right),
$$


where $\left\{j_{1}, \ldots, j_{n}\right\}=\left\{j \in I \mid \beta_{j} \neq b^{(j)}\right\}$. Suppose $\beta_{j}=b^{(j)}$. Then $\mathrm{e}_{j}\left(\beta_{j}\right)=$ $\mathrm{e}_{j}\left(b^{(j)}\right)$, but

$$
\mathrm{e}_{j}\left(b^{(j)}\right)=\left(\left(b^{(j)}\right)^{* *} ;\left(1, \ldots, 1, b^{(j)}, 1, \ldots, 1\right)\right)=\left(\left(b^{(j)}\right) ;\left(1, \ldots, 1, b^{(j)}, 1, \ldots, 1\right)\right)
$$

and

$$
\left(\left(b^{(j)}\right) ;\left(1, \ldots, 1, b^{(j)}, 1, \ldots, 1\right)\right) \geq\left(b ;\left(b^{(i)}\right)\right)=\boldsymbol{\beta}^{* *},
$$

so these terms cancel out from $\bigwedge_{i \in I} \mathrm{e}_{i}\left(\beta_{i}\right)$ when forming the meet with $\boldsymbol{\beta}^{* *}$.

Theorem 4.10. $\mathbf{P}$ is a $\mathbf{P C S}$-free product of the p-semilattices $\mathbf{S}_{i}(i \in I)$.

Proof. We will show that $\mathbf{P}$ is a $\mathbf{P C S}$-free product of its subalgebras $\mathbf{S}_{i}^{\prime}(i \in I)$. To this end, let $\mathbf{T}$ be an arbitrary p-semilattice together with a family of p-semilattice homomorphisms $t_{i}^{\prime}: \mathbf{S}_{i}^{\prime} \rightarrow \mathbf{T}$ for $i \in T$. We will construct a p-semilattice homomorphism $t: \mathbf{P} \rightarrow \mathbf{T}$ extending $t_{i}^{\prime}$ for all $i \in I$.

Note that $t_{i}^{\prime} \uparrow \mathbf{B}_{i}^{\prime}$ is a Boolean homomorphism from $\mathbf{B}_{i}^{\prime}$ to $S k(\mathbf{T})$, so there exists a Boolean homomorphism $t_{B}^{\prime}: \mathbf{B}^{\prime} \rightarrow S k(\mathbf{T})$ extending $\left.t_{i}^{\prime}\right\rceil \mathbf{B}_{i}^{\prime}$ for all $i \in I$ as $\mathbf{B}^{\prime}$ is a $\mathbf{B A}$-free product of its subalgebras $\mathbf{B}_{i}^{\prime}$. Consider $\boldsymbol{\beta} \in P$ where $\boldsymbol{\beta}=\left(b ;\left(\beta_{i}\right)\right)$. By Corollary 4.9 , we have $\boldsymbol{\beta}=\boldsymbol{\beta}^{* *} \wedge \bigwedge_{i \in I} \mathrm{e}_{i}\left(\beta_{i}\right)$.

Define $t(\boldsymbol{\beta}):=t_{B}^{\prime}\left(\boldsymbol{\beta}^{* *}\right) \wedge \bigwedge_{i \in I} t_{i}^{\prime}\left(\mathrm{e}_{i}\left(\beta_{i}\right)\right)$. We have to show that $t$ is compatible with $\wedge$ and ${ }^{*}$.

Starting with meet, consider $\boldsymbol{\alpha}=\left(a ;\left(\alpha_{i}\right)\right)$ and $\boldsymbol{\beta}=\left(b ;\left(\beta_{i}\right)\right)$ in $\mathbf{P}$. We note first that

$$
\begin{aligned}
(\boldsymbol{\alpha} \wedge \boldsymbol{\beta})^{* *} & =\left(a \wedge b ;\left((a \wedge b)^{(i)}\right) \leq\left((a \wedge b)^{(i)} ;\left(1, \ldots, 1,(a \wedge b)^{(i)}, 1, \ldots, 1\right)\right)\right. \\
& =\mathrm{e}_{i}\left((a \wedge b)^{(i)}\right) .
\end{aligned}
$$

Since $\mathrm{e}_{i}\left((a \wedge b)^{(i)}\right) \in \mathbf{B}_{i}^{\prime}$, this implies $t_{i}^{\prime}\left(\mathrm{e}_{i}\left((a \wedge b)^{(i)}\right)\right)=t_{B}^{\prime}\left(\mathrm{e}_{i}\left((a \wedge b)^{(i)}\right)\right)$ and thus

$$
t_{B}^{\prime}\left((\boldsymbol{\alpha} \wedge \boldsymbol{\beta})^{* *}\right) \leq t_{i}^{\prime}\left(\mathrm{e}_{i}\left((a \wedge b)^{(i)}\right)\right)
$$

for all $i \in I$. Now compute

$$
\begin{aligned}
t(\boldsymbol{\alpha}) \wedge t(\boldsymbol{\beta}) & =t_{B}^{\prime}\left(\boldsymbol{\alpha}^{* *}\right) \wedge \bigwedge_{i \in I} t_{i}^{\prime}\left(\mathrm{e}_{i}\left(\alpha_{i}\right)\right) \wedge t_{B}^{\prime}\left(\boldsymbol{\beta}^{* *}\right) \wedge \bigwedge_{i \in I} t_{i}^{\prime}\left(\mathrm{e}_{i}\left(\beta_{i}\right)\right) \\
& =t_{B}^{\prime}\left((\boldsymbol{\alpha} \wedge \boldsymbol{\beta})^{* *}\right) \wedge \bigwedge_{i \in I}\left(t_{i}^{\prime}\left(\mathrm{e}_{i}\left(\alpha_{i}\right)\right) \wedge t_{i}^{\prime}\left(\mathrm{e}_{i}\left(\beta_{i}\right)\right)\right)
\end{aligned}
$$

which, by (4.1),

$$
\begin{aligned}
& =t_{B}^{\prime}\left((\boldsymbol{\alpha} \wedge \boldsymbol{\beta})^{* *}\right) \wedge \bigwedge_{i \in I}\left(t_{i}^{\prime}\left(\mathrm{e}_{i}\left(\alpha_{i}\right)\right) \wedge t_{i}^{\prime}\left(\mathrm{e}_{i}\left(\beta_{i}\right)\right) \wedge t_{i}^{\prime}\left(\mathrm{e}_{i}\left((a \wedge b)^{(i)}\right)\right)\right) \\
& =t_{B}^{\prime}\left((\boldsymbol{\alpha} \wedge \boldsymbol{\beta})^{* *}\right) \wedge \bigwedge_{i \in I} t_{i}^{\prime}\left(\mathrm{e}_{i}\left(\alpha_{i} \wedge \beta_{i} \wedge(a \wedge b)^{(i)}\right)\right) \\
& =t(\boldsymbol{\alpha} \wedge \boldsymbol{\beta})
\end{aligned}
$$

since the $\left.\alpha_{i} \wedge \beta_{i} \wedge(a \wedge b)^{(i)}\right)$ are the components of $\boldsymbol{\alpha} \wedge \boldsymbol{\beta}$.

For pseudocomplements, put $\boldsymbol{\alpha}=\boldsymbol{\beta}$ in (3) to obtain $t_{B}^{\prime}\left(\boldsymbol{\alpha}^{* *}\right) \leq t_{i}^{\prime}\left(\mathrm{e}_{i}\left(a^{(i)}\right)\right)$ and observe that $t_{i}^{\prime}\left(\mathrm{e}_{i}\left(a^{(i)}\right)\right)=t_{i}^{\prime}\left(\mathrm{e}_{i}\left(\alpha_{i}^{* *}\right)\right)=\left(t_{i}^{\prime}\left(\mathrm{e}_{i}\left(\alpha_{i}\right)\right)^{* *}\right.$, thus

$$
t_{B}^{\prime}\left(\boldsymbol{\alpha}^{* *}\right) \leq\left(t_{i}^{\prime}\left(\mathrm{e}_{i}\left(\alpha_{i}\right)\right)^{* *}\right.
$$


for all $i \in I$. With that, we obtain

$$
(t(\boldsymbol{\alpha}))^{* *}=\left(t_{B}^{\prime}\left(\boldsymbol{\alpha}^{* *}\right)\right)^{* *} \wedge \bigwedge_{i \in I}\left(t_{i}^{\prime}\left(\mathrm{e}_{i}\left(\alpha_{i}\right)\right)\right)^{* *}=\left(t_{B}^{\prime}\left(\boldsymbol{\alpha}^{* *}\right)\right)^{* *}=t_{B}^{\prime}\left(\boldsymbol{\alpha}^{* *}\right)
$$

by (4.2) (distributing ${ }^{* *}$ over $\bigwedge$ is in order since the meet may be replaced by a finitary one, see the proof of Corollary 4.9). Forming pseudocomplements once more, we get $t(\boldsymbol{\alpha})^{*}=\left(t_{B}^{\prime}\left(\boldsymbol{\alpha}^{* *}\right)\right)^{*}=t_{B}^{\prime}\left(\boldsymbol{\alpha}^{*}\right)$.

If $\boldsymbol{\alpha} \in \mathbf{B}^{\prime}$, putting $\boldsymbol{\alpha}=\boldsymbol{\beta}$ in (4.1) results in $t_{B}^{\prime}(\boldsymbol{\alpha})=t_{B}^{\prime}\left(\boldsymbol{\alpha}^{* *}\right) \leq t_{i}^{\prime}\left(\mathrm{e}_{i}\left(a^{(i)}\right)\right)=$ $t_{B}^{\prime}\left(\mathrm{e}_{i}\left(a^{(i)}\right)\right)$, and thus $t(\boldsymbol{\alpha})=t_{B}^{\prime}\left(\boldsymbol{\alpha}^{* *}\right) \wedge \bigwedge_{i \in I} t_{B}^{\prime}\left(\mathrm{e}_{i}\left(a^{(i)}\right)\right)=t_{B}^{\prime}(\boldsymbol{\alpha})$. It follows that $t \uparrow \mathbf{B}^{\prime}=t_{B}^{\prime}$. We conclude that $t\left(\boldsymbol{\alpha}^{*}\right)=t_{B}^{\prime}\left(\boldsymbol{\alpha}^{*}\right)$, and since also $t(\boldsymbol{\alpha})^{*}=t_{B}^{\prime}\left(\boldsymbol{\alpha}^{*}\right)$ as seen above, we obtain $t(\boldsymbol{\alpha})^{*}=t\left(\boldsymbol{\alpha}^{*}\right)$ as desired.

It remains to show that $t$ extends $t_{j}^{\prime}$ for all $j \in I$. Consider $\mathrm{e}_{j}(\xi) \in \mathbf{S}_{j}$. Remembering that $(\boldsymbol{\alpha})_{i}$ stands for the $i$-th component of $\boldsymbol{\alpha}$, compute $t\left(\mathrm{e}_{j}(\xi)\right)=$ $t_{B}^{\prime}\left(\left(\mathrm{e}_{j}(\xi)^{* *}\right)\right) \wedge \bigwedge_{i \in I} t_{i}^{\prime}\left(\mathrm{e}_{i}\left(\left(\mathrm{e}_{j}(\xi)\right)_{i}\right)\right)$. Now $t_{B}^{\prime}\left(\left(\mathrm{e}_{j}(\xi)^{* *}\right)\right)=t_{j}^{\prime}\left(\left(\mathrm{e}_{j}(\xi)\right)^{* *}\right)$ because $\left(\mathrm{e}_{j}(\xi)^{* *}\right) \in \mathbf{B}_{j}^{\prime}$ and $t_{B}^{\prime}$ extends $t_{j}^{\prime} \uparrow \mathbf{B}_{j}^{\prime}$. Since $\left(\mathrm{e}_{j}(\xi)\right)_{i}=1$ for $i \neq j$ and $\left(\mathrm{e}_{j}(\xi)\right)_{j}=\xi$, we have $t_{i}^{\prime}\left(\mathrm{e}_{i}\left(\left(\mathrm{e}_{j}(\xi)\right)_{i}\right)\right)=1$ for $i \neq j$ and $t_{j}^{\prime}\left(\mathrm{e}_{j}\left(\left(\mathrm{e}_{j}(\xi)\right)_{j}\right)\right)=$ $t_{j}^{\prime}\left(\mathrm{e}_{j}(\xi)\right)$. Hence $t\left(\mathrm{e}_{j}(\xi)\right)=t_{B}^{\prime}\left(\left(\mathrm{e}_{j}(\xi)^{* *}\right)\right) \wedge \bigwedge_{i \in I} t_{i}^{\prime}\left(\mathrm{e}_{i}\left(\left(\mathrm{e}_{j}(\xi)\right)_{i}\right)\right)$ reduces to $t_{j}^{\prime}\left(\left(\mathrm{e}_{j}(\xi)\right)^{* *}\right) \wedge t_{j}^{\prime}\left(\mathrm{e}_{j}(\xi)\right)$ which equals $t_{j}^{\prime}\left(\mathrm{e}_{j}(\xi)\right)$, and we are done.

\section{Free p-semilattices}

Free p-semilattices were first described in [5] and subsequently in Balbes [2] and, of particular interest here, [8] and [9], using different approaches. Another description results in [6] from specializing the free product construction given there. Here, we show that the construction obtained in Section 4 specializes to the one given in [8].

Let $\mathbf{F}_{\mathbf{P C S}}(I)$ be the p-semilattice freely generated by a set $\left\{g_{i} \mid i \in I\right\}$ of free generators. It is immediate that $\mathbf{F}_{\mathbf{P C S}}(\emptyset) \cong \mathbf{2}$, the 2-element p-semilattice, and $\mathbf{F}_{\mathbf{P C S}}(\{i\}) \cong \mathbf{N}_{\mathbf{5}}$, the p-semilattice reduct of the pentagon realized as $\left\{0, g_{i}, g_{i}^{*}, g_{i}^{* *}, 1\right\}$. Hence, $\mathbf{F}_{\mathbf{P C S}}(I) \cong \coprod_{\mathbf{P C S}}\left\{\left(\mathbf{N}_{\mathbf{5}}\right)_{i} \mid i \in I\right\}$, the free product of $|I|$ many copies of $\mathbf{N}_{\mathbf{5}}$. By Proposition 2.2, we have

$$
S k\left(\mathbf{F}_{\mathbf{P C S}}(I)\right) \cong \coprod_{\mathbf{B A}}\left\{\left(\mathbf{2}^{2}\right)_{i} \mid i \in I\right\} \cong \mathbf{F}_{\mathbf{B A}}(I),
$$

the Boolean algebra freely generated by $|I|$ many generators.

So let $\mathbf{B}=\mathbf{F}_{\mathbf{B A}}(I)$ and select, w.l.o.g., $\left\{g_{i}^{* *} \mid i \in I\right\}$ as its set of free generators. For any $0 \neq b \in B$ and $i \in I$, we have $b^{(i)} \in\left\{g_{i}^{*}, g_{i}^{* *}, 1_{i}\right\}$, with $b^{(i)} \neq 1_{i}$ for at most finitely many $i \in I$. Conversely, let $I_{\text {fin }} \subseteq I$ be any finite subset of $I$ and consider $\left(b_{i}\right)_{i \in I}$ with $b_{i} \in\left\{g_{i}^{*}, g_{i}^{* *}\right\}$ for $i \in I_{f i n}$ and $b_{i}=1_{i}$ for $i \notin I_{\text {fin }}$. It is easy to describe the elements $b \in B$ satisfying $b^{(i)}=b_{i}$ for all $i \in I$ : Obviously $0 \neq b$ and thus $0<b^{(i)}$, hence $b \leq b^{(i)} \in\left\{g_{i}^{*}, g_{i}^{* *}, 1_{i}\right\}$. It follows that $b$ has the required property if and only if $b \leq g_{i}^{*}$, respectively $b \leq g_{i}^{* *}$, for $i \in I_{\text {fin }}$ and $b \not \leq g_{i}^{*}$ as well as $b \not \leq g_{i}^{* *}$ for $i \notin I_{\text {fin }}$. By Fact 3.2, $b$ is of this type if and only if it has a disjunctive normal form where exactly 
the $g_{i}^{*}$, respectively the $g_{i}^{* *}$, with $i \in I_{\text {fin }}$ occur as common meetands in all meet terms.

Passing to $\mathbf{F}_{\mathbf{P C S}}(I)$, we analyze its structure by describing the Glivenko $\operatorname{class} \Gamma(\boldsymbol{\beta}) \subseteq \mathbf{F}_{\mathbf{P C S}}(I)$ for each $0 \neq \boldsymbol{\beta} \in S k\left(\mathbf{F}_{\mathbf{P C S}}(I)\right)=\mathbf{B}^{\prime} \cong \iota(\mathbf{B})$. In this case, $\boldsymbol{\beta}$ has the form $\boldsymbol{\beta}=\left(b ;\left(b^{(i)}\right)\right)$ with $b \in B$ and such that $\left.b^{(i)}\right) \in\left\{g_{i}^{*}, g_{i}^{* *}\right\}$ for some finite subset $I_{\text {fin }} \subseteq I$ and $\left.b^{(i)}\right)=1_{i}$ for $i \notin I_{\text {fin }}$. Consider $\boldsymbol{\alpha}=$ $\left(a ;\left(\alpha_{i}\right)\right) \in \mathbf{F}_{\mathbf{P C S}}(I)$ such that $\boldsymbol{\alpha}^{* *}=\boldsymbol{\beta}$. It follows that $\boldsymbol{\alpha}$ may be obtained from $\boldsymbol{\beta}$ by replacing some or all of the occurrences of $g_{i}^{* *}$ in $\boldsymbol{\beta}$ by $g_{i}$. Let $\mu(\boldsymbol{\beta})$ be the number of $g_{i}^{* *}$ 's occurring in $\boldsymbol{\beta}$. Then $\Gamma(\boldsymbol{\beta})$ is isomorphic, in its induced order, to $2^{\mu(\beta)}$, the Boolean lattice with $\mu(\boldsymbol{\beta})$ atoms. Summing up, we have

Theorem 5.1. As an ordered set, $\mathbf{F}_{\mathbf{P C S}}(I) \backslash\{0\}$ is isomorphic to

$$
\left\{\boldsymbol{\beta}=\left(b ;\left(\beta_{i}\right)\right) \mid 0 \neq b \in \mathbf{F}_{\mathbf{B A}}(I), b^{(i)}=\beta_{i}^{* *} \in\left\{g_{i}^{*}, g_{i}^{* *}\right\}\right.
$$

for at most finitely many $i \in I$, and $\beta_{i}=1_{i}$ elsewhere, under the componentwise order. For such $\boldsymbol{\beta}$, one has $\Gamma(\boldsymbol{\beta})=\Gamma\left(\boldsymbol{\beta}^{* *}\right) \cong \mathbf{2}^{\mu\left(\beta^{* *}\right)}$. Explicitly, $\Gamma(\boldsymbol{\beta})$ is the set of all $\iota(b) \wedge \boldsymbol{\xi}$ where $\boldsymbol{\xi}$ is a finite meet of elements $\mathrm{e}_{i}\left(g_{i}\right)$, respectively $\mathrm{e}_{i}\left(g_{i}^{* *}\right)$, (if $\left.b^{(i)}=g_{i}^{* *}\right)$ and $\mathrm{e}_{i}\left(g_{i}^{*}\right)$ (if $\left.b^{(i)}=g_{i}^{*}\right)$. Conversely, given $\left(\beta_{i}\right)$ with $\beta_{i}^{* *} \in\left\{g_{i}^{*}, g_{i}^{* *}\right\}$ for at most finitely many $i \in I$ and $\beta_{i}=1_{i}$ elsewhere, then $\Gamma\left(\left(b ;\left(\beta_{i}\right)\right)\right)$ has this form exactly if $b \in \mathbf{F}_{\mathbf{B A}}(I)$ satisfies $b \leq g_{i}^{* *} \Leftrightarrow \beta_{i}^{* *}=g_{i}^{* *}$ and $b \leq g_{i}^{*} \Leftrightarrow \beta_{i}^{* *}=g_{i}^{*}$.

This complements the description of $\mathbf{F}_{\mathbf{P C S}}(I)$ given in [8].

\section{REFERENCES}

[1] Adams, M.E., Schmid, J.: Pseudocomplemented semilattices are finite-to-finite relatively universal. Algebra Universalis 58, 303-333(2008)

[2] Balbes, R.: On free pseudo-complemented and relatively pseudo-complemented semi-lattices. Fund. Math. 78, 119-131 (1973)

[3] Chajda, I., Halaš, R., Kühr, J.: Semilattice Structures. Heldermann Verlag, Lemgo (2007)

[4] Grätzer, G.: Universal Algebra, 2nd edn. Springer, New York (1979)

[5] Jones, G.T.: Pseudo-Complemented Semi-Lattices. PhD thesis, UCLA (1974)

[6] Katriňák, T., Heleyová, Z.: Free Products of Pseudocomplemented Semilattices. Semigroup Forum 60, 450-469 (2000)

[7] Koppelberg, S.: Handbook of Boolean Algebras, vol. 1. North Holland, Amsterdam (1989)

[8] Schmid, J.: On the structure of free pseudocomplemented semilattices. Houston J. Math. 16, 71-85 (1990)

[9] Schmid, J.: Addenda. Houston J. Math. 16, 301 (1990)

[10] Sikorski, R.: Boolean Algebras, 3rd edn. Springer, New York (1969)

\section{E. AdAmS}

Department of Mathematics, State University of New York, New Paltz, NY 12561, USA e-mail: adamsm@newpaltz.edu

\section{JÜRG SCHMID}

Institute of Mathematics, University of Bern, CH-3012 Bern, Switzerland e-mail: juerg.schmid@math.unibe.ch 